Article

\title{
Twinning Phenomena along and beyond the Bain Path
}

\author{
Sandra Kauffmann-Weiss ${ }^{1,2,3, *}$, Alexander Kauffmann ${ }^{1,2}$, Robert Niemann ${ }^{1}$, \\ Jens Freudenberger ${ }^{1,4}$, Ludwig Schultz ${ }^{1,2}$ and Sebastian Fähler ${ }^{1,3}$ \\ ${ }^{1}$ IFW Dresden, P.O. Box 270116, 01171 Dresden, Germany; \\ E-Mails: a.kauffmann@ifw-dresden.de (A.K.); r.niemann@ifw-dresden.de (R.N.); \\ j.freudenberger@ifw-dresden.de (J.F.); 1.schultz@ifw-dresden.de (L.S.); \\ s.faehler@ifw-dresden.de (S.F.) \\ 2 TU Dresden, Institute of Materials Science, 01062 Dresden, Germany \\ 3 TU Chemnitz, Institute of Physics, 09107 Chemnitz, Germany \\ ${ }^{4}$ TU Bergakademie Freiberg, Institute of Materials Science, 09596 Freiberg, Germany \\ * Author to whom correspondence should be addressed; E-Mail: s.weiss@ifw-dresden.de; \\ Tel.: +49-351-4659-665; Fax: +49-351-4659-541.
}

Received: 24 July 2013; in revised form: 11 September 2013 / Accepted: 26 September 2013 /

Published: 9 October 2013

\begin{abstract}
Twinning is a phenomenon that occurs, e.g., during deformation, martensitic transformation and film growth. The present study shows that the crystallography of twinning can be described by two twinning modes along the complete Bain transformation path and beyond connecting body-centered and face-centered cubic structures. To probe this concept, we used strained epitaxial films of the Fe-Pd magnetic shape memory system. As the substrate acts as an absolute reference frame, we could show by pole figure measurements that all observed twinning can be a body-centered and face-centered cubic twinning mode. This continuously transforms towards identity when approaching the complementary structure.
\end{abstract}

Keywords: deformation twinning; transformation twinning; adaptive twinning; magnetic shape memory; Fe-Pd 


\section{Introduction}

In the field of physical metallurgy, materials, such as shape memory alloys, high-strength materials, high-temperature alloys and multiferroics, exhibit different kinds of twinning phenomena, which have a strong influence on the functional properties. The twin formation occurs during different processes [1], like deformation [2-4], film growth [5], recovery and/or recrystallization [6], phase transformation [7] or adaptive nanotwinning [8].

In the present investigation, we combine experiments and simulations of several twinning phenomena observed within the binary Fe-Pd system. Fe-Pd has been suggested as a model system for martensitic transformations and twinning, already in 1938 [9]. The disordered ferromagnetic shape memory alloy Fe-Pd exhibits a martensitic transformation that follows the concept of the Bain path for deformation of a unit cell from face-centered cubic (fcc) to body-centered cubic (bcc) [10,11]. For example, the magnetic shape memory alloy $\mathrm{Fe}_{70} \mathrm{Pd}_{30}$ shows such a transformation during cooling. In addition, by changing the Pd content from 25 to 33 at.\% and by quenching from temperatures above 1,123 K, the following metastable phases occur in bulk single crystals at room temperature: bcc, bct (body-centered tetragonal), fct (face-centered tetragonal) and fcc phase [11]. At the atomic scale, the necessary orientation relation is given by the Bain path methodology [12] and visualized in Figure 1. Regarding a face-centered atom of the austenitic fcc unit cell (dark grey shaded atoms) as the corner of the bct unit cell (bright grey shaded atoms), this bet unit cell is tetragonal instead of cubic and aligned along the $\langle 110\rangle$ directions of the fcc unit cell. This highlights the key feature of the Bain path-a simplified description of the transformation using a single parameter: the tetragonal distortion reflected by the $c /\left.a\right|_{\text {fct }}$-ratio. For a Fe-Pd system, this one parameter is sufficient, as the volume remains almost constant [13].

Figure 1. Scheme of the elementary twin reactions along the Bain path: (a) fcc (facecentered cubic) system (dark grey atoms) with $\{111\}_{\mathrm{fcc}}\langle 11 \overline{2}\rangle_{\mathrm{fcc}}$ and (b) bcc (body-centered cubic) system (bright grey atoms) with $\{112\}_{\text {bcc }}\langle 11 \overline{1}\rangle_{\text {bcc }}$ twinning mode. Common lattice planes are shaded in grey.

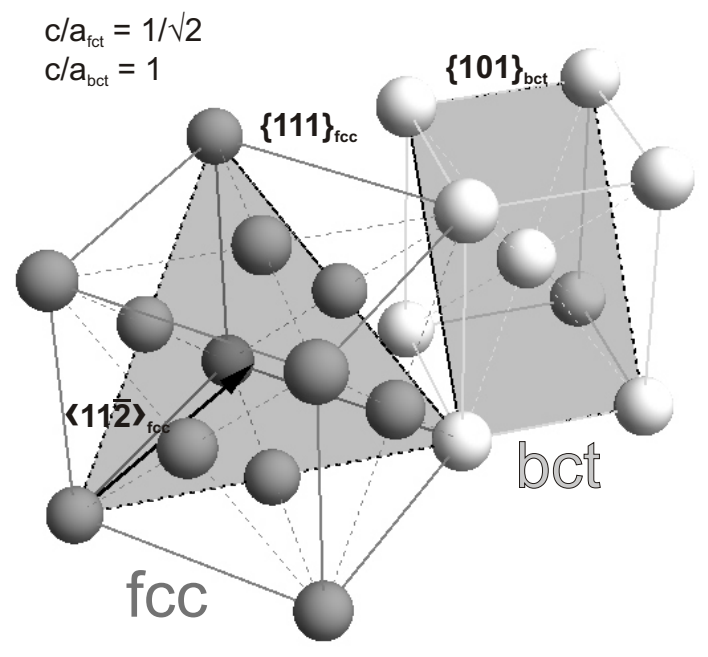

(a)

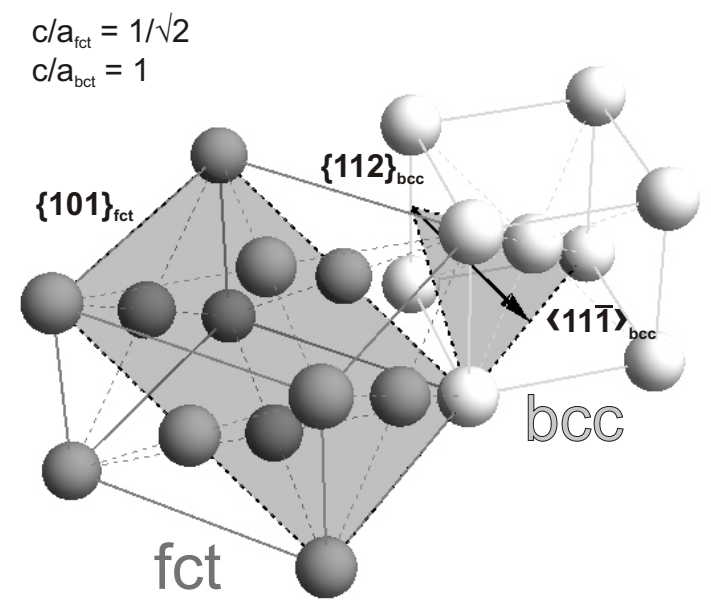

(b) 
There are several definitions for twinning in the literature. For a discussion of the different definitions, see [14]. In the present work, twinning is defined as follows: the win and matrix can be brought to congruence by applying a crystallographic symmetry operation (reflection, inversion and rotation) that does not belong to the crystallography of the phase, and the boundaries between the variants are called twin boundaries.

Usually, the basic properties of twin formation are discussed for cubic fcc and bcc metals. The most common twinning modes appearing in fcc and bcc metals are $\{111\}_{\mathrm{fcc}}\langle 11 \overline{2}\rangle_{\mathrm{fcc}}$ and $\{112\}_{\mathrm{bcc}}\langle 11 \overline{1}\rangle_{\mathrm{bcc}}$, respectively $[2,3]$. The elements of this notation correspond to twinning planes $\{\ldots\}$ and shear directions $\langle\ldots\rangle$. Since the normal of the twinning planes, as well as their shear direction (in Figure 1) are rational, they are called compound twins [15]. The orientation transformation that connects the original variant and the twinned variant can be expressed by either a rotation of $180^{\circ}$ about the twin plane normal or by a rotation of $180^{\circ}$ about the shear direction. The advantage for those compound twins is that the crystal orientation can be obtained either by a mirror operation or by a $180^{\circ}$ rotation. According to this general description and the notation given in Figure 1, the elementary twin reaction can be extended to be valid along the Bain path, which continuously connects the fcc to the bcc lattice by a tetragonal distortion [12,16]. The corresponding twin elements are $\{111\}_{\mathrm{fct}}\langle 11 \overline{2}\rangle_{\mathrm{fct}}$ and $\{112\}_{\text {bct }}\langle 11 \overline{1}\rangle_{\text {bct }}$.

For the experiments shown here, we used $\mathrm{Fe}_{100-x} \mathrm{Pd}_{x}$ films with different structures. These different structures are described by the tetragonal distortion of the unit cell. According to Bain's concept, we use in this paper the lattice parameters, $a_{\mathrm{fct}}$ and $c_{\mathrm{fct}}$, of the fct unit cell to describe all different structures. An alternative expression to the fct unit cell is the bct unit cell. The fct description is easily converted to the bct description by multiplying with a conversion factor of $\sqrt{2}$. For epitaxial $\mathrm{Fe}_{70} \mathrm{Pd}_{30}$ films, the $c /\left.a\right|_{\text {fct }}$-ratio can be changed continuously from $c /\left.a\right|_{\text {fct }}=1.0$ for fcc to $c /\left.a\right|_{\text {fct }}=1 / \sqrt{2}$ for bcc. Close to this composition, the energy for straining the unit cell is relatively low, and all tetragonal distortions along the Bain path can be stabilized in thin films [13]. Due to the essentially flat energy landscape even beyond the limits of the Bain path, $\mathrm{Fe}_{70} \mathrm{Pd}_{30}$ films can be strained with $c /\left.a\right|_{\text {fct }}>1.0$ [17].

The substrate as an absolute reference frame allows for analyzing of the structure and the orientation relationship using pole figure measurements and comparing these results to simulations of different twinning modes. This paper is arranged as follows. At the beginning, we describe a concept for all twinning modes along the Bain path. After a short description of the background on the simulations, we characterize in detail the different modes of deformation twinning (Sections 5.1 and 5.2). A second focus is on the proof of transformation twins in annealed films (Section 5.3). Finally, we experimentally provide the adaptive nanotwinning in highly strained Fe-Pd films (Section 5.4).

\section{Experimental Section}

Deposition at ambient temperatures allows for increasing of the critical film thickness, as the introduction of misfit dislocations is a thermally activated process [18]. It is favorable that the Fe-Pd system exhibits a structural instability and a softening of the crystal lattice near room temperature [19]. Therefore, epitaxial Fe-Pd films can be produced with different methods, like pulsed laser deposition [20], molecular beam epitaxy [21] or sputter deposition [13]. Here, we use the possibility of different preparation routes to easily change film thickness and composition. 
$\mathrm{Fe}_{100-\mathrm{x}} \mathrm{Pd}_{\mathrm{x}}$ films were deposited at room temperature on $\mathrm{MgO}(001)[100]$ single crystalline substrates using pulsed laser deposition (PLD) from elemental targets [20]. The sequence from bcc to fct was observed for as-deposited films with different Pd contents (25-33 at.\%Pd). Following the procedure of bulk samples [11], the pulsed laser-deposited $\mathrm{Fe}_{100-\mathrm{x}} \mathrm{Pd}_{\mathrm{x}}$ films were annealed in a quartz tube at $1273 \mathrm{~K}$ for $10 \mathrm{~min}$ under vacuum conditions $\left(<10^{-5} \mathrm{mbar}\right)$ and water quenched afterwards. After this heat treatment, the same metastable phases as in bulk samples were obtained [22].

To produce faster and thicker epitaxial Fe-Pd films, DC magnetron sputtering was used. $\mathrm{Fe}_{70} \mathrm{Pd}_{30}$ films were deposited from an alloy target on different epitaxially-grown metallic buffer layers (e.g., $\mathrm{Cu}$ and $\mathrm{Cr}$ ) at room temperature (base pressure $<10^{-8} \mathrm{mbar}$ ) [13,17]. The $50 \mathrm{~nm} \mathrm{Cr}$ buffer layer was deposited at $300{ }^{\circ} \mathrm{C}$ on $\mathrm{MgO}(001)[100]$ single crystalline substrates. When $50 \mathrm{~nm} \mathrm{Cu}$, exhibiting a fcc lattice, is used as the buffer, prior to deposition, an adhesion layer of $5 \mathrm{~nm} \mathrm{Cr}$ was grown on the $\mathrm{MgO}$ substrate. The deposition temperature of the $\mathrm{Cu}$ buffer layer was room temperature.

The sample surface was examined using a scanning electron microscope (SEM) from Zeiss (Leo 1530 Gemini). Using energy dispersive X-ray spectroscopy (EDX) and a $\mathrm{Fe}_{70} \mathrm{Pd}_{30}$ standard, the composition of the film was determined. Pole figures revealing the global texture were measured using a four circle setup (Phillips X'Pert, $\mathrm{Cu}-\mathrm{K}_{\alpha}$ radiation) in the range of $0^{\circ} \leq \varphi \leq 360^{\circ}$ and $\psi \leq 80^{\circ}$ in steps of $1^{\circ}$.

\section{Twinning Modes along the Bain Path}

While for an exactly cubic fcc structure, the bcc twinning mode does not occur as it transforms the crystal into its identity, for a tetragonally distorted fct lattice $\left(c /\left.a\right|_{\text {fct }}<1.0\right)$, this is not the case anymore. The $\{101\}_{\mathrm{fct}}\langle\overline{1} 01\rangle_{\mathrm{fct}}$ twinning mode for a fct structure is, according to Figure 1, equivalent to $\{112\}_{\text {bct }}\langle 11 \overline{1}\rangle_{\text {bct }}$ twinning. In analogy, one can describe the twinning system of a tetragonally distorted bct structure either by $\{101\}_{\text {bct }}\langle\overline{1} 01\rangle_{\text {bct }}$ or $\{111\}_{\text {fct }}\langle 11 \overline{2}\rangle_{\text {fct }}$.

Thus, the concept of the Bain path allows for the describing of both deformation and transformation twins by the same concept. Moreover, with this intuitive description, one can compare the energies of both twins. The shape change resulting from the shear leads to elastic distortions, and with that, the strain energy increases. Given by [23,24], for both cases, the strain energy, $E$, is proportional to the square of the shear, $s$. For $f c c$ deformation, twinning is $E=(c / r) \mu s^{2}$, and for transformation twinning, $E=(c / r) \mu\left(s^{2}+\delta^{2}\right)$, Where $\mu$ is the shear modulus of the parent lattice, $c / r$ the thickness-to-length ratio of the twins and $\delta$ the dilatational component normal to the habit plane. To minimize the energy, the twins should be long and thin. It is established that the elastic energy and the interface energy compete in a twinned material [25]. The shear energy associated with transformation twinning increases squared with the tetragonal distortion, as expected from the linear elastic theory, and thus, a deformation twin is expected to have a much larger excess energy. To understand twinning, one has also to consider the strain, which can be compensated for by the particular twinning mode. This is much lower for a slight tetragonal distortion compared to deformation twinning.

While for a material with a cubic structure deformation, twinning is the only alternative to elastic deformation, for a slightly tetragonally distorted martensitic material, two stages are possible. At low strains, first the transformation twin boundary is moved. Once this mechanism is finished (e.g., in a single variant state), deformation twinning can occur to adapt the strain. For huge tetragonal distortions, 
somewhere in the middle of the Bain path, both twinning mechanism are expected to degenerated and may occur simultaneously.

\section{Simulation}

\subsection{Simulation of fcc and bcc Twins}

According to Bain's concept and with respect to the reference system (MgO substrate), we can simulate all different twin modes using the following basics. The congruent description of the twinning elements [16] within the tetragonal systems leads to the analogousness of:

$$
\{111\}_{\mathrm{fct}}\langle 11 \overline{2}\rangle_{\mathrm{fct}} \equiv\{101\}_{\text {bct }}\langle 10 \overline{1}\rangle_{\text {bct }} \text { as well as }\{112\}_{\text {bct }}\langle 11 \overline{1}\rangle_{\text {bct }} \equiv\{101\}_{\text {fct }}\langle 10 \overline{1}\rangle_{\text {fct }}
$$

Thus, in the border systems, the opposed twin reactions transform the crystal into its identity. Namely, the fcc-type twin reaction $\{111\}_{\mathrm{fct}}\langle 11 \overline{2}\rangle_{\mathrm{fct}}$ coincidences with the two-fold rotation axis of the bcc system, as well as the bcc-type twin reaction $\{112\}_{\text {bct }}\langle 11 \overline{1}\rangle_{\text {bct }}$ coincidences with two-fold rotation axis of the fcc system. The associated shears, $s$, of the twins as functions of $c /\left.a\right|_{\text {fct }}$ or $c /\left.a\right|_{\text {bct }}$ are shown in Figure 2. These are:

$$
\begin{aligned}
& s_{\{111\}_{\mathrm{fct}}\langle 11 \overline{2}\rangle_{\mathrm{fct}}}=\frac{\left|1-2\left(c / a_{\mathrm{fct}}\right)^{2}\right|}{\sqrt{2} c / a_{\mathrm{fct}}} \\
& s_{\{112\}_{\mathrm{bct}}\langle 11 \overline{1}\rangle_{\mathrm{bct}}}=\frac{\left|\left(c / a_{\mathrm{bct}}\right)^{2}-2\right|}{\sqrt{2} c / a_{\mathrm{bct}}}
\end{aligned}
$$

which correctly yield to $1 / \sqrt{2}$ in the border systems and vanishing shear in the opposed border systems.

Figure 2. The associated shear of fcc-type $\{111\}_{\mathrm{fct}}\langle 11 \overline{2}\rangle_{\mathrm{fct}}$ and bcc-type $\{112\}_{\mathrm{bct}}\langle 11 \overline{1}\rangle_{\mathrm{bct}}$ twins as functions of $c / a_{\mathrm{fct}}$ or $c / a_{\mathrm{bct}}$.

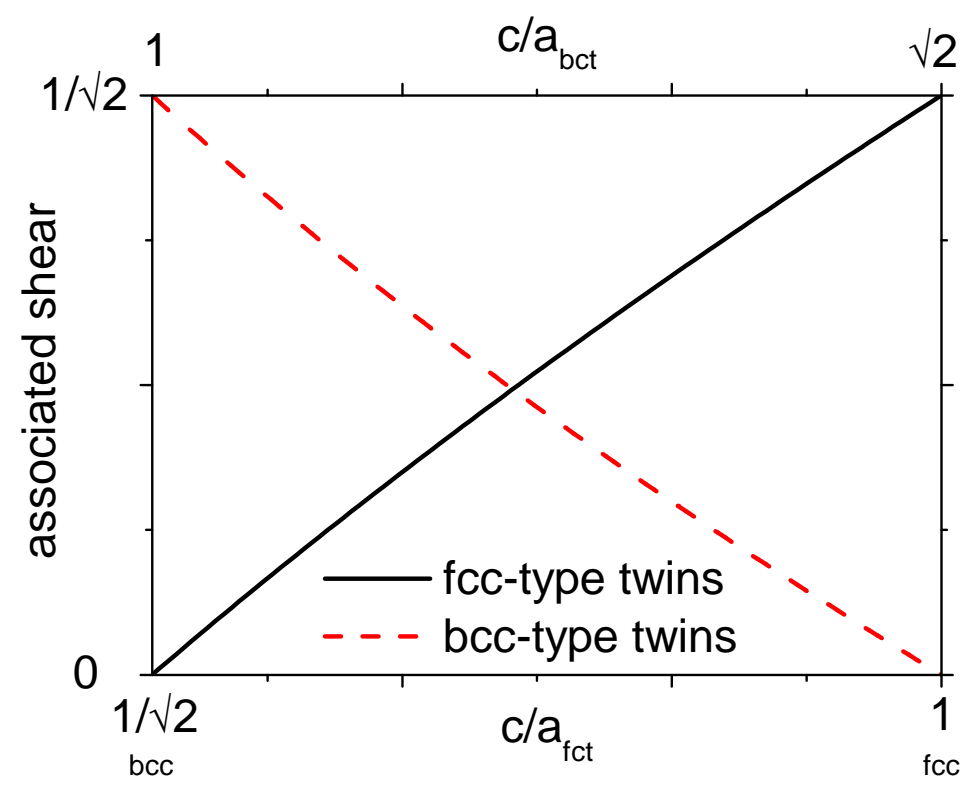

For the investigation of orientation changes associated with twins of different kinds, we will discuss, among others, $\{111\}_{\mathrm{fct}}$ or $\{101\}_{\text {bct }}$ pole figures of thin films. By using the rotation transformation 
described in Section 1, the rotation matrices for the different twin systems can be developed. The corresponding twin variant orientations are calculated by applying these rotation matrices to the basic coordinate system of the matrix. The term, matrix, refers to the epitaxially-grown part of the film. Thus, reflections are given by the vectors of the plane, $(h k l)$, with respect to the $\mathrm{MgO}$ substrate. The first and second generation of twins are visualized by red circles and blue triangles, respectively. The terms, first and second generation, of twins is used to describe a hierarchical microstructure [25] and a twin-within-twins microstructure [26,27]. The effect of bcc-type twinning $\{112\}_{\text {bct }}\langle 11 \overline{1}\rangle_{\text {bct }}$ and fcc-type twinning $\{111\}_{\mathrm{fct}}\langle 11 \overline{2}\rangle_{\mathrm{fct}}$ on $\{111\}_{\mathrm{fct}}$ is summarized in Figure 3 for a single crystal of the fcc- and bcc-border systems. Due to the four-fold symmetry of the (001) matrix, it is sufficient to show only one quadrant. According to the above-mentioned details, bcc-type twinning does not alter the atomic positions for a fcc matrix. Hence, the positions of matrix and twin variants coincide (Figure $3 b$ ).

Figure 3. Pole figure simulation of bcc-type $\{112\}_{\text {bct }}\langle 11 \overline{1}\rangle_{\text {bct }}(\mathbf{a}, \mathbf{b})$ and fcc-type $\{111\}_{\mathrm{fct}}\langle 11 \overline{2}\rangle_{\mathrm{fct}}$ twins (c,d) for the limits of the Bain path at the bcc and the fcc state.



\subsection{Simulation of Martensitic Twins}

The Bain concept cannot describe a martensitic transformation, since a phase boundary between a cubic and a distorted structure is not considered. This problem can be solved using the Phenomenological Theory of Martensite Crystallography (PMTC) [7]. Here, we briefly describe how to simulate pole figures of a twinned structure formed during a martensitic transformation using this theory. The martensitic transformation is of the first order and requires a propagating phase front (habit plane), where austenite and martensite have to be compatible, i.e., the elastic energy at the interface has to be minimal. In the following calculation, an adaption of the twinned martensite to the parent austenite and, 
therefore, a rotation as a consequence of the straining of the lattice is considered. Following PTMC, the transformation is expressed by a $3 \times 3$ matrix $T=Q U$, where $Q$ describes the rotation and $U$ the stretching of the unit cell. A scalar multiplication of the transformation matrix, $T$, with a lattice vector, $a_{i}$, of the austenite yields a corresponding vector in a martensitic variant, $v_{i}$.

$$
\overrightarrow{v_{i}}=T \overrightarrow{a_{i}}=Q U \overrightarrow{a_{i}}
$$

In the present case, the cubic to tetragonal transformation is given by the symmetric matrices [7]:

$$
U_{1}=\left(\begin{array}{ccc}
\frac{c_{m}}{a_{p}} & 0 & 0 \\
0 & \frac{a_{m}}{a_{p}} & 0 \\
0 & 0 & \frac{a_{m}}{a_{p}}
\end{array}\right)
$$

and:

$$
U_{2}=\left(\begin{array}{ccc}
\frac{a_{m}}{a_{p}} & 0 & 0 \\
0 & \frac{c_{m}}{a_{p}} & 0 \\
0 & 0 & \frac{a_{m}}{a_{p}}
\end{array}\right)
$$

$U_{1}$ and $U_{2}$ correspond to two in-plane variants ( $c_{m}$ about parallel to the substrate surface) of martensite. In the present experiments, only these two martensitic variants are observed, and thus, variants with the $c$-axis out-of-plane were omitted in the simulated pole figures for clarity.

The condition of elastic compatibility is fulfilled when the transformation matrix $T$ (and, consequently, also, the corresponding $U$ ) has a middle eigenvalue equal to one, which means that the lattice remains unstrained in the direction of the corresponding eigenvector. In general, $U_{1}$ and $U_{2}$ do not have a middle eigenvalue of one, and for this reason, none of the variants can form a martensite-austenite interface on its own. Instead, the system introduces twin boundaries, which means that it forms an austenite-martensite interface using different variants that are compatible with the interface on average. These variants are connected to each other by the twinning condition:

$$
Q U_{2}-U_{1}=\vec{a} \otimes \vec{n}
$$

which expresses that one variant $\left(U_{2}\right)$ is rotated using the matrix, $Q$, to form a stress-free twin-boundary with another variant $\left(U_{1}\right)$. The twin boundary is then defined by the twin shear vector, $\vec{a}$, and twin plane normal, $\vec{n}$.

The habit plane condition:

$$
Q^{\prime}\left(\lambda Q U_{2}+(1-\lambda) U_{1}=I+\vec{b} \otimes \vec{m}\right.
$$

introduces a parameter $\lambda$ for the volume fraction of variants and rotates the entire twinned microstructure using the rotation matrix, $Q^{\prime}$, to form a stress-free habit plane. The habit plane is defined by the habit plane shear vector, $\vec{b}$, and habit plane normal, $\vec{m}$. Since both processes, twinning and habit plane formation, happen simultaneously, it is more demonstrative to re-write both equations using the definitions $Q=Q_{2}{ }^{-1} Q_{1}$ and $Q^{\prime}=Q_{2}$ to obtain the form:

$$
Q_{2}{ }^{-1} Q_{1} U_{2}-U_{1}=\vec{a} \otimes \vec{n}
$$


for the twinning condition and:

$$
\lambda Q_{1} U_{2}+(1-\lambda) Q_{2} U_{1}=I+\vec{b} \otimes \vec{m}
$$

for the habit plane condition.

These equations have to be solved for the rotation $Q_{1}$ and $Q_{2}$ to obtain the orientation of all martensitic variants that fulfill these conditions. $I$ is the identity matrix. The value $\lambda$ and the vectors, $\vec{a}, \vec{n}, \vec{b}$ and $\vec{m}$, are also obtained during the solution, which is described elsewhere in great detail [7].

Since the twinning condition has two solutions and the habit plane condition four solutions per twin, eight unique solutions exist when only in-plane variants are taken into account. Finally, each solution contains two martensitic variants, given by their sets of lattice vectors. The corresponding reflections of planes $(h k l)$ are calculated again with respect to the $\mathrm{MgO}$ substrate using Equation (3). Note that the matrices, $U_{1}$ and $U_{2}$ (and, accordingly, the lattice parameters of austenite and martensite) are the only parameters necessary to calculate the variant orientation.

\section{Results and Discussion}

\section{1. fcc Deformation/Growth Twins}

Previous study on as-deposited $\mathrm{Fe}_{57} \mathrm{Pd}_{43}$ films on $\mathrm{MgO}$ substrates prepared with PLD showed that only films with a thickness up to $\approx 20 \mathrm{~nm}$ grow completely epitaxially [20]. Stress measurements have shown large stresses up to $700 \mathrm{MPa}$ for thick Fe-Pd films [28]. The formation of deformation twins reduces the elastic energy originating from stress at the expense of a comparably low interface energy of the twin boundaries. For a $300 \mathrm{~nm}$ thick as-deposited $\mathrm{Fe}_{57} \mathrm{Pd}_{43}$ film, a fcc structure is expected from the phase diagram. An SEM picture of the surface in Figure 4 shows a fine twinned microstructure that is rotated by $45^{\circ}$ with respect to the $\mathrm{MgO}\langle 100\rangle$ edges. This morphology agreed with the assumption of fine long twins from the strain energy (Section 3). X-ray structure analysis revealed that the unit cell is slightly distorted with $c /\left.a\right|_{\mathrm{fct}}=0.97$, and the $c$-axis is aligned in-plane along $\mathrm{MgO}[100]$ and/or $[010]$.

Figure 4. SEM picture of an as-deposited $\mathrm{Fe}_{57} \mathrm{Pd}_{43}$ film on a $\mathrm{MgO}$ substrate. The edges of the pictures are parallel to $\mathrm{MgO}\langle 100\rangle$. On the film surface, a fine twinned structure with $45^{\circ}$ to $\mathrm{MgO}\langle 100\rangle$ edges is observed, which is shown schematically in the inset.

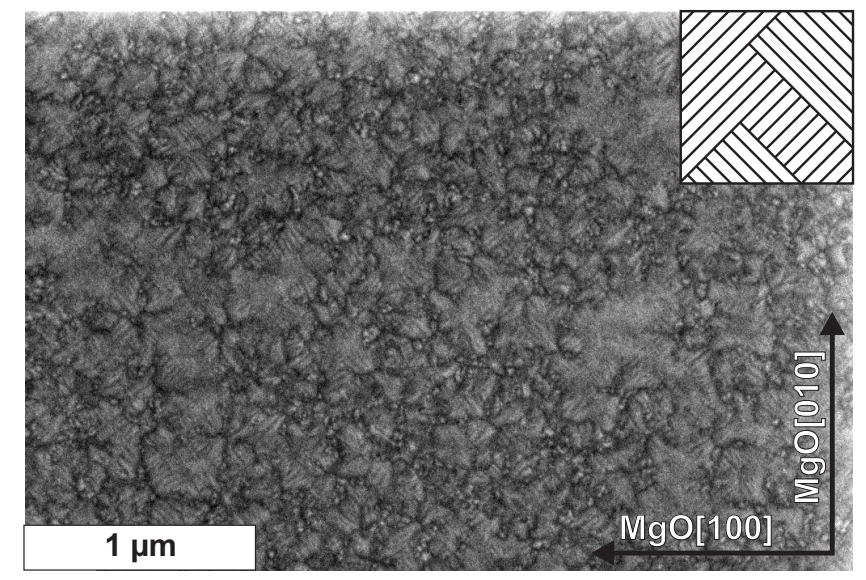


Using $\{111\}_{\mathrm{fct}},\{202\}_{\mathrm{fct}},\{200\}_{\mathrm{fct}}$ and $\{311\}_{\mathrm{fct}}$ pole figures, the film texture and orientation relationship were investigated in detail (Figure 5). Here, all four pole figures can be measured, since the intensity of the Fe-Pd reflections are high enough, and no overlapping with the substrate occurs. In addition to the major intensities (black squares) originating from the epitaxially-grown part, further intensities were observed in all pole figures. These additional intensities have lower counts compared to the major intensities. By comparing the measured (right quadrant) with a simulated $\{111\}_{\text {fct }}$ pole figure (left quadrant), the additional intensities can be explained by $\{111\}_{\mathrm{fct}}\langle 11 \overline{2}\rangle_{\mathrm{fct}}$ deformation/growth twins. The simulation of the first twin generation (all possible solutions) agree on number and positions. The small splitting of the first twin generation is caused by two possible alignments of the $c$-axes-along $\mathrm{MgO}[100]$ and [010] direction-and of the slightly tetragonal distorted lattice with $c /\left.a\right|_{\text {fct }}=0.97$. For a perfect cubic structure $\left(c /\left.a\right|_{\text {fct }}=1\right)$, no difference between both orientations would occur. A further stress relaxation during film growth is achieved by twinning of the second and, if necessary, higher generations. In the present case, a second generation occurs, which is partly visible in Figure 5a.

Figure 5. Comparison of the measured pole figure of the $\mathrm{Fe}_{57} \mathrm{Pd}_{43}$ film (right quadrant) with simulations of fcc-type $\{111\}_{\text {fct }}\langle 11 \overline{2}\rangle_{\text {fct }}$ twins (left quadrant) of (a) $\{111\}_{\text {fct }} ;$ (b) $\{202\}_{\text {fct }}$; (c) $\{200\}_{\text {fct }}$ and (d) $\{311\}_{\text {fct }}$. Black squares represent the epitaxial matrix, red circles the first twin generation and the blue triangles, the second twin generation.

a) $\{111\}_{\text {fot }}$ pole figure

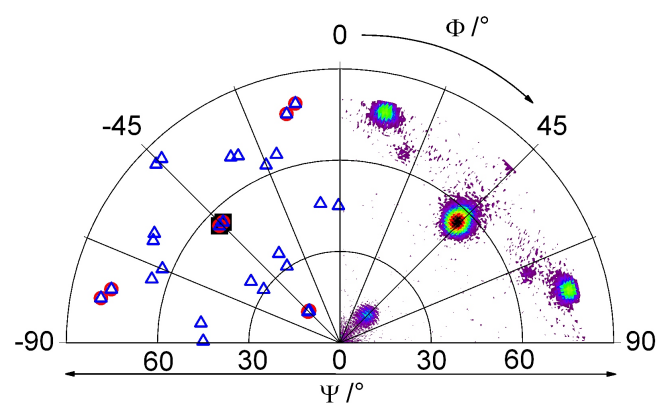

c) $\{200\}_{\text {ftt }}$ pole figure

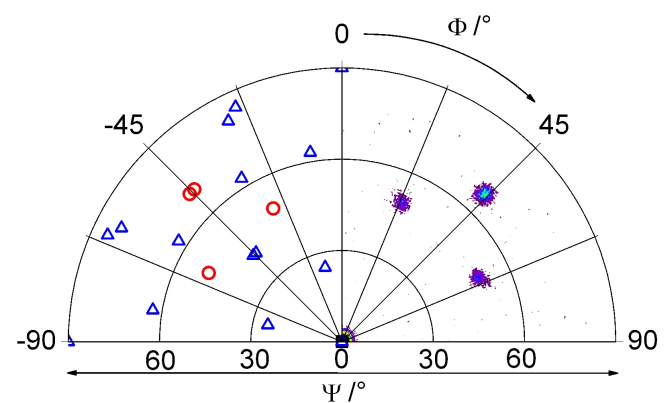

b) $\{202\}_{\mathrm{fct}}$ pole figure

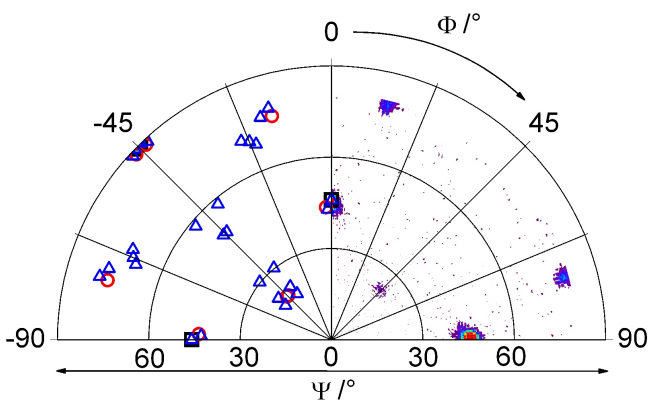

d) $\{311\}_{\text {fot }}$ pole figure

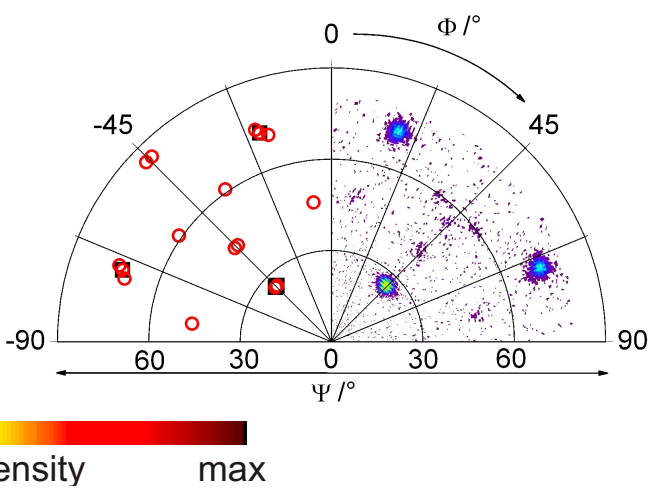

\section{2. bcc Deformation/Growth Twins}

A $\mathrm{Fe}_{70} \mathrm{Pd}_{30}$ film on an additional $\mathrm{Cr}$ buffer exhibits a bct structure with $c /\left.a\right|_{\text {fct }}=0.77$ and the $c$-axis out-of-plane [13] and the $a$-axes parallel to $\mathrm{MgO}[100]$ and $\mathrm{MgO}[010]$. Those epitaxial films have a flat surface without any features. For a $700 \mathrm{~nm}$ thick $\mathrm{Fe}_{70} \mathrm{Pd}_{30}$ film, additional pyramidal features are visible 
at the surface (Figure 6). The mid-rib of these features is parallel to the $\mathrm{MgO}[100]$ or [010] edges and $\approx 600 \mathrm{~nm}$ long and $\approx 80 \mathrm{~nm}$ high. This triangular form looks similar, like the so-called indentions in [29], which occur during deformation twinning in bcc metals. Although, these pyramidal features also appear like a herring bone structure, which is often observed in a martensitic wedge. However, for a unique decision between these different explanations, a detailed transmission electron microscopic study would be required.

Figure 6. SEM picture of a $\mathrm{Fe}_{70} \mathrm{Pd}_{30}$ on a $\mathrm{Cr}$ buffer layer. The edges of the pictures are parallel to $\mathrm{MgO}\langle 100\rangle$. On the surface, pyramidal features parallel to $\mathrm{MgO}\langle 100\rangle$ are visible.

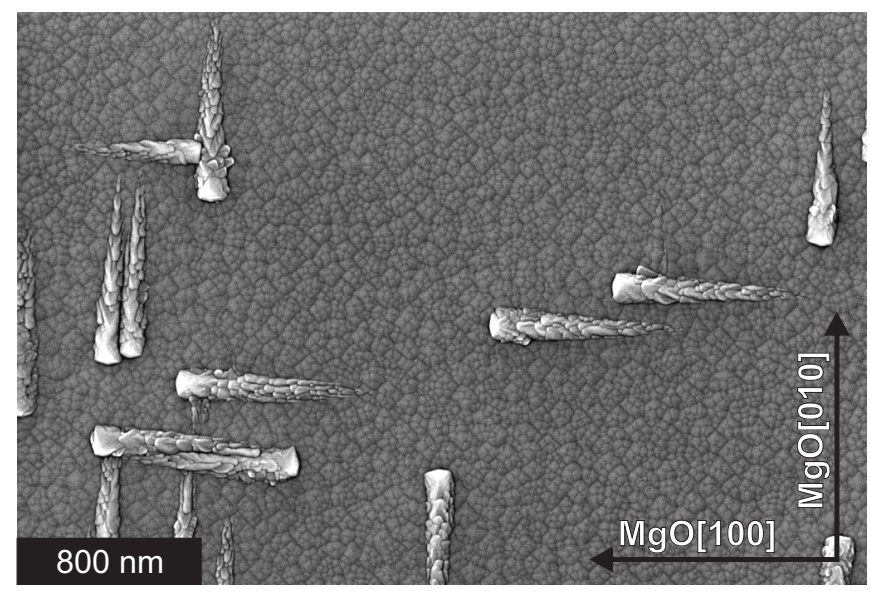

With a tetragonal distortion of $c /\left.a\right|_{\mathrm{fct}}=0.77$, the Fe-Pd unit cell is close to the bcc structure. According to Bain's concept, we still continue with the fct notation. Pole figure measurements of $\{111\}_{\text {fct }},\{002\}_{\text {fct }}$ and $\{311\}_{\text {fct }}$ reflections reveal that the observations in the SEM are accompanied by a bcc-type twinned microstructure (Figure 7). In the present case, we show only these three pole figures, since $\{220\}_{\text {fct }}$ overlaps with peaks of the Cr layer. Besides the major intensities of the epitaxial matrix, further intensities of variants are visible and can be explained by the first generation of $\{112\}_{\text {bcc }}\langle 11 \overline{1}\rangle_{\text {bcc }}$ twins. Analogous to the fcc-type twin mode, all possible solutions are shown by the circles (left quadrant).

Figure 7. Comparison of measured pole figure of a $\mathrm{Fe}_{70} \mathrm{Pd}_{30}$ film on $\mathrm{Cr}$ buffer (right quadrant) with simulations of bcc-type $\{112\}_{\text {bct }}\langle 11 \overline{1}\rangle_{\text {bct }}$ twins (left quadrant) of (a) $\{111\}_{\text {fct }}$; (b) $\{002\}_{\mathrm{fct}}$ and (c) $\{311\}_{\mathrm{fct}}$. Black squares represent the epitaxial matrix and the red circles, the first twin generation.

a) $\{111\}_{\mathrm{fct}}$ pole figure

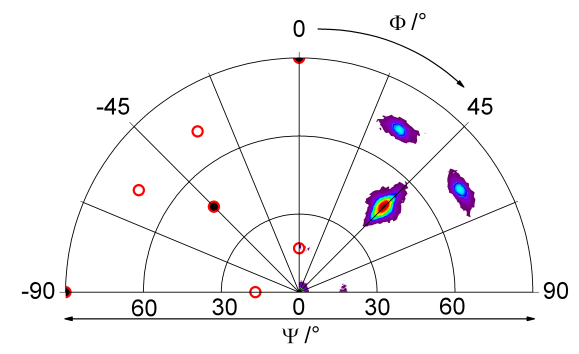

b) $\{002\}_{\text {fct }}$ pole figure



c) $\{311\}_{\text {ftt }}$ pole figure

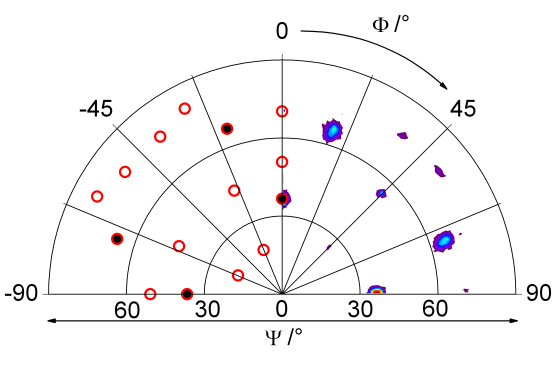


In comparison to thick $\mathrm{Fe}_{70} \mathrm{Pd}_{30}$ films grown on another buffer material, like $\mathrm{Au}$, there is a huge difference in film growth. $\mathrm{Fe}_{70} \mathrm{Pd}_{30}$ films grown on $\mathrm{Au}$ buffer have a tetragonal distortion of $c /\left.a\right|_{\text {fct }}=0.79$ that is similar to distortions of $\mathrm{Fe}_{70} \mathrm{Pd}_{30}$ films on $\mathrm{Cr}$ buffer. The misfit between the unit cells of $\mathrm{Fe}_{70} \mathrm{Pd}_{30}$ and $\mathrm{Au}$ or $\mathrm{Cr}$ is $-0.5 \%$ or $-0.3 \%$, respectively. The only essential difference between both buffer materials is that $\mathrm{Cr}$ exhibits a bcc structure and $\mathrm{Au}$, a fcc structure.

Density functional theory (DFT) calculations reveal that a bcc structure of Fe-Pd $\left(c /\left.a\right|_{\text {fct }}=0.71\right.$ ) is the energetically favored structure along the Bain path [30]. Close to this bcc ground state, $\mathrm{Fe}_{70} \mathrm{Pd}_{30}$ films on Au buffer grow epitaxial up to a thickness of $1.2 \mu \mathrm{m}$ [31]. In case of this Au buffer, no bcc-type twinning was observed. At higher thicknesses, a continuous defect accumulation occurs, resulting in a relaxation towards a polycrystalline bcc structure. We suggest that these differences originate from the fact that twinning is a collective phenomenon, and thus, nucleation is required. A buffer of the same structure, which presumably already exhibits a few bcc-grown twins, may facilitate twinning. In contrast to that, an fcc buffer is expected to exhibit only incompatible fcc-type twins.

\subsection{Transformation Twins}

Here, we present the consequences of a heat treatment $(1,273 \mathrm{~K}$ for $10 \mathrm{~min})$ on the texture for a $\mathrm{Fe}_{75} \mathrm{Pd}_{25}$ film on $\mathrm{MgO}$ substrate. In the as-deposited state, the film exhibits a bcc structure $\left(c /\left.a\right|_{\text {fct }}=0.71\right)$. After annealing, a change from a cubic bcc (002) texture to a bct structure with a (200) texture and $c /\left.a\right|_{\mathrm{fct}}=0.77$ was observed [22].

Figure 8. SEM picture (Backscatterd electron contrast) of a twinned microstructure obtained for an annealed $\mathrm{Fe}_{75} \mathrm{Pd}_{25}$ film. The edges of the picture are parallel to $\mathrm{MgO}\langle 100\rangle$. The inset shows the corresponding FFT and two different angles are visible $\left(13^{\circ}\right.$ and $\left.30^{\circ}\right)$, which originate from two different twin modes.
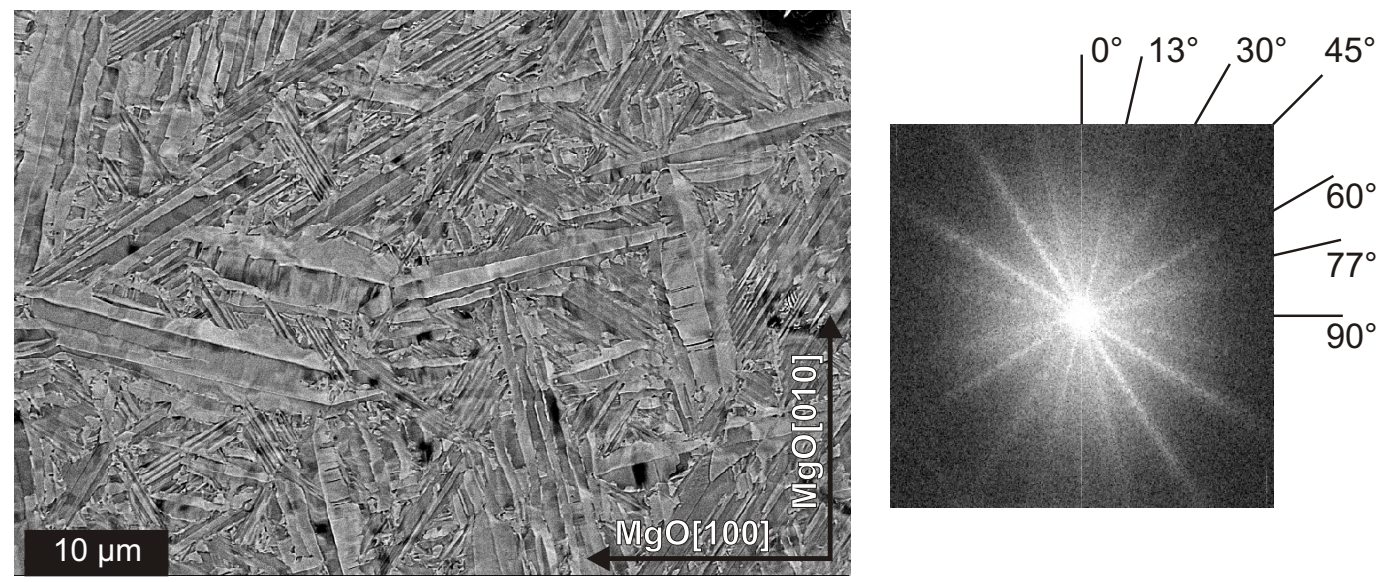

Figure 8 shows a twinned microstructure of the film surface after annealing. Most of the twins are orientated close to the $\mathrm{MgO}\langle 110\rangle$ directions. These fine twins appear to be similar to $\{101\}_{\mathrm{fct}}$ transformation twins in Fe-Pd bulk materials [11,32] and thick Ni-Mn-Ga films [27]. In addition, wide twins aligned with a deviation of $\approx 13^{\circ}$ from the $\mathrm{MgO}\langle 100\rangle$ edges are observed. These irregular twins do not exhibit sharp interfaces to neighboring twin variants and appear more similar to bct-type deformation 
or annealing twins [11]. The twin bands are comparably large and contain a mid-rib along their center part. Both ends exhibit characteristic ends for bcc-type deformation twins, which are so-called indentations [2]. The corresponding fast Fourier transform (FFT) picture (inset in Figure 8) shows the different angles that can occur from both transformation and deformation twinning. The FFT picture exhibits four-fold symmetry that originates from the epitaxial growth on the (001) $\mathrm{MgO}$ substrate and not from the symmetry of the martensitic phase. However, it cannot be completely excluded that these are twins of a higher generation in a twin-within-twin microstructure, as observed in Ni-Mn-Ga [8,26].

To validate the formed microstructure and the variant orientation after annealing, $\{111\}_{\mathrm{fct}},\{311\}_{\mathrm{fct}}$, $\{200\}_{\mathrm{fct}}$ and $\{220\}_{\mathrm{fct}}$ pole figures were measured. During annealing, the symmetry of the film according to the phase diagram is expected to be fcc. After cooling, the austenite reversibly transforms into the fct martensite and at lower temperatures, irreversible into the bct martensite. During this martensitic transformation, the unit cells of the martensite have to adapt to the fcc austenite at the habit plane by tilting and rotation.

Figure 9. Comparison of measured pole figure of an annealed $\mathrm{Fe}_{75} \mathrm{Pd}_{25}$ film (right quadrant) with simulations of martensitic $\{101\}_{\mathrm{fct}}\langle\overline{1} 01\rangle_{\mathrm{fct}}$ twins for $c /\left.a\right|_{\mathrm{fct}}=0.77$ (left quadrant) of (a) $\{111\}_{\mathrm{fct}}$; (b) $\{311\}_{\mathrm{fct}} ;$ (c) $\{200\}_{\mathrm{fct}}$; and (d) $\{220\}_{\mathrm{fct}}$.

a) $\{111\}_{\text {fot }}$ pole figure

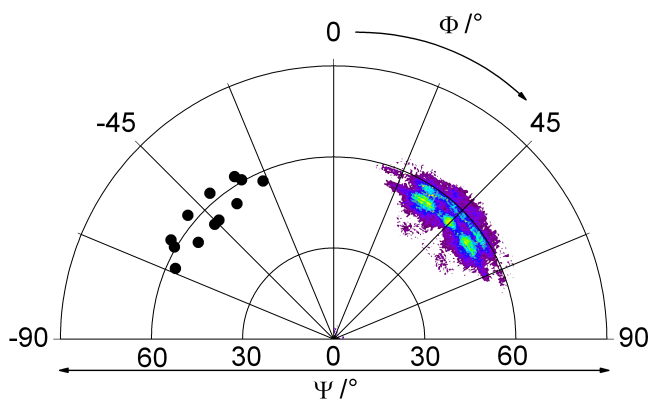

c) $\{200\}_{\text {fot }}$ pole figure

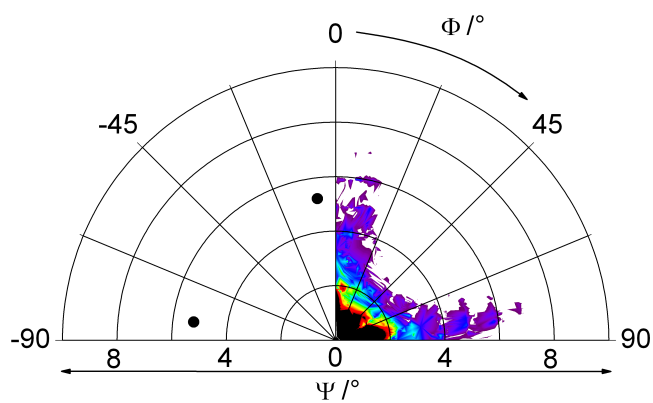

b) $\{311\}_{\text {fct }}$ pole figure

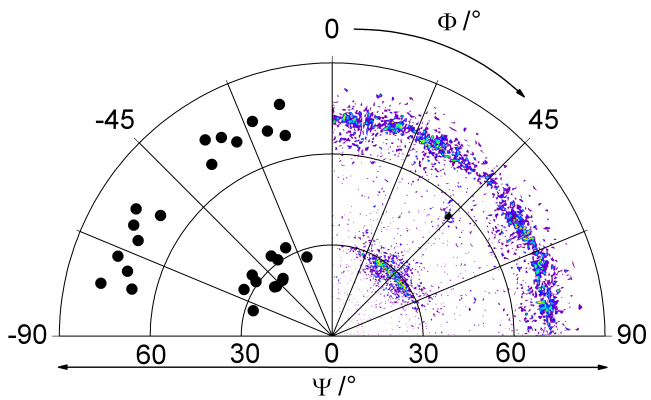

d) $\{220\}_{\text {ftt }}$ pole figure

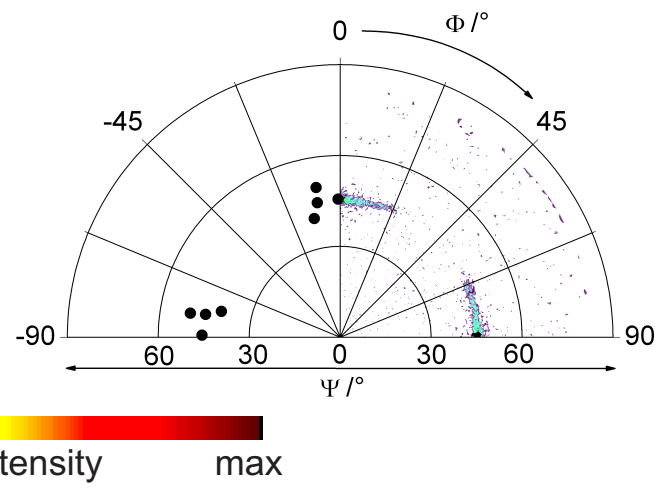

In Figure 9, the results of the simulation are compared with the measured pole figures. As described in Section 4, \{101 $\}_{\text {fct }}\langle\overline{1} 01\rangle_{\text {fct }}$ transformation twins can be calculated using the phenomenological theory of martensite. The transformation from a high symmetry to a low symmetry phase is related to a change in the lattice, here, from cubic ( $a_{p}=0.3776 \mathrm{~nm}$ [11]) to tetragonal $\left(a_{m}=0.4069 \mathrm{~nm}\right.$ and $c_{m}=0.3133 \mathrm{~nm}$ from XRD). During this transformation, differently-oriented twin variants of the low symmetry structure are possible. We observed that only martensitic variants with their short $c$-axis in-plane are formed. For 
the case with the $c$-axis out-of-plane, further intensities should occur at additional $\Psi$ and $\Phi$ values: e.g., in the $\{111\}_{\text {fct }}$ pole figure at about $\Psi \approx 40^{\circ}$. Since this is not the case in the present investigation, we do not show the solutions for twin boundaries originating from variants with their $c$-axis out-of-plane. A variant selection is often observed in thin martensitic films of reduced dimension compared to bulk. Often, the mechanism can be attributed to stress [33].

The comparison of the measured and simulated pole figures reveals a good agreement with respect to the positions of the intensities. The smearing of the measured intensities may have two reasons: Firstly, the disordered Fe-Pd alloy exhibits a structural instability, resulting in a softening of the crystal structure [19]. While in common materials, this is guaranteed by a deep minimum of free energy, the particular Fe-Pd system exhibits an essentially flat double well potential [13]. The flat energy landscape is intimately connected with the martensitic transformation of Fe-Pd. This behavior can allow a certain variation of the tetragonal distortion at finite temperatures and with that, the associated angle $\Psi$ in the pole figures. However, the peak width observed from X-ray in Bragg-Brentano geometry in [22] is relatively small and suggests less variation of the $c /\left.a\right|_{\text {fct }}$-ratio. Secondly, a reason for the smearing might lie within the existence of small angle grain boundaries. This assumption is proven by the SEM picture of the film surface shown in Figure 8. Besides twin variants with an alignment close to the $\mathrm{MgO}\langle 110\rangle$ edges, variants with slightly higher or lower inclinations are observed. Furthermore, a change of the twin mechanism from transformation to deformation twinning can occur and leads to a broadening of the intensities.

\subsection{Adaptive Nanotwins}

Recently, it has been shown that introducing $\mathrm{Cu}$ as a buffer layer causes the unit cell of Fe-Pd and its alloy, $\mathrm{Fe}-\mathrm{Pd}-\mathrm{Cu}$, to be highly strained beyond the limits of the Bain path [17,34]. The tetragonal distortion of the parent unit cell can reach values up to $c /\left.a\right|_{\text {fct }}=1.09$ with a preferred out-of-plane orientation of the $c$-axis. In DFT calculations of the energy landscape as a function of the tetragonal distortion, a second minimum was observed close to these experimental values $[17,34]$. Instead of an expected strong increase in energy beyond the Bain path, these supercell calculations show a further minimum of energy, due to the formation of $\{101\}_{\text {fct }}$ twin boundaries perpendicular to the substrate. These twin boundaries have a very low energy and can thus reduce the elastic energy in highly strained films. Therefore, straining beyond the Bain path opens a new relaxation mechanism, where a single variant with $c /\left.a\right|_{\text {fct }}>1$ spontaneously transforms to a nanotwinned structure with $c /\left.a\right|_{\text {fct }}<1$, exhibiting a lower energy, since they are closer to the global minimum at bcc $\left(c /\left.a\right|_{\mathrm{fct}}=0,71\right)$.

This relaxation process is similar to the formation of an adaptive phase occurring, e.g., in $\mathrm{Ni}-\mathrm{Mn}-\mathrm{Ga}$ [8,35]. However, in the present work, the habit plane is defined by the artificial (001) film-buffer interface. Adaptive nanotwinning is only possible when starting beyond the Bain path. It is a rare case of a diffusionless transformation between two tetragonal structures, where no reduction of symmetry is necessary. This is only possible with a geometrically-defined twinning relation. The $c /\left.a\right|_{\text {fct }}$-ratio of the nanotwins adjust to the boundary conditions of the parent unit cell by the following geometrically relation [17].

$$
\left.\frac{c}{a}\right|_{\text {fct }}=\sqrt{\frac{1}{2}+\frac{1}{2}\left(\left.\frac{c}{a}\right|_{\text {Twins }}\right)^{-2}}
$$


For the nanotwinned structure, the $c$-axis is in-plane (Figure 10b) and $c /\left.a\right|_{\text {twin,fct }}=0.88$. Figure 10a summaries the measured and the calculated $\{111\}_{\text {fct }}$ pole figures using $\{101\}_{\text {fct }}$ twin boundaries perpendicular to the substrate for the matrix with the in-plane $c$-axis. The other pole figures give similar results and are, thus, not shown here: the theoretical positions of the twinned variants occur at a $\Psi=90^{\circ}$, where we are not able to measure, or overlap with the reflections of the $\mathrm{Cu}$ buffer. The intensities at $\Phi=45^{\circ}$ and $\Psi=57^{\circ}$ arise from the epitaxially-grown part with the $c$-axis out-of-plane and agree with the highest counts in the measured pole figure (right quadrant). Close to this, two additional intensities appear, resulting from two possible aligned $c$-axes in-plane and the tetragonal distorted unit cell. These orientations lead to a weak smearing out of the central intensity of the matrix. The twin variants from each aligned matrix (c-axis along the $\mathrm{MgO}[100]$ or $\mathrm{MgO}[010]$ direction, Figure 10b) are marked with red circles and agree with the measured positions.

Figure 10. (a) Comparison of the measured $\{111\}_{\text {fct }}$ pole figure of a $\mathrm{Fe}_{70} \mathrm{Pd}_{30}$ film on $\mathrm{Cu}$ buffer (right quadrant) to a simulation of this pole figure, including $\{101\}_{\text {fct }}$ nanotwins (left quadrant). Black squares represent the epitaxial matrix and red circles, the first twin generation. (b) Schematic sketch for the twin configuration.

a)

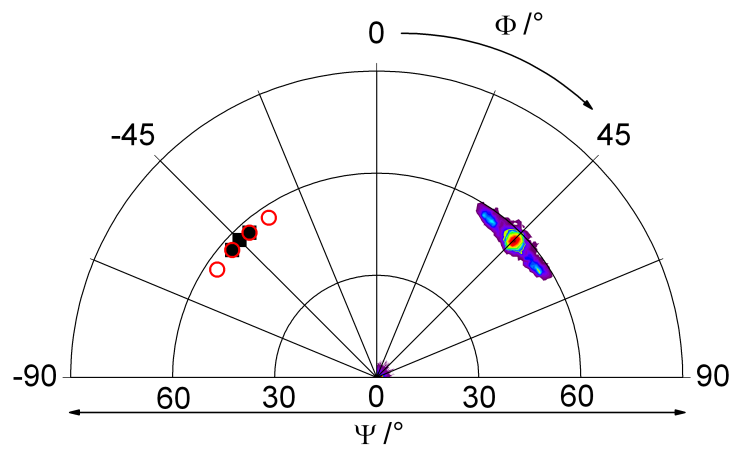

b)

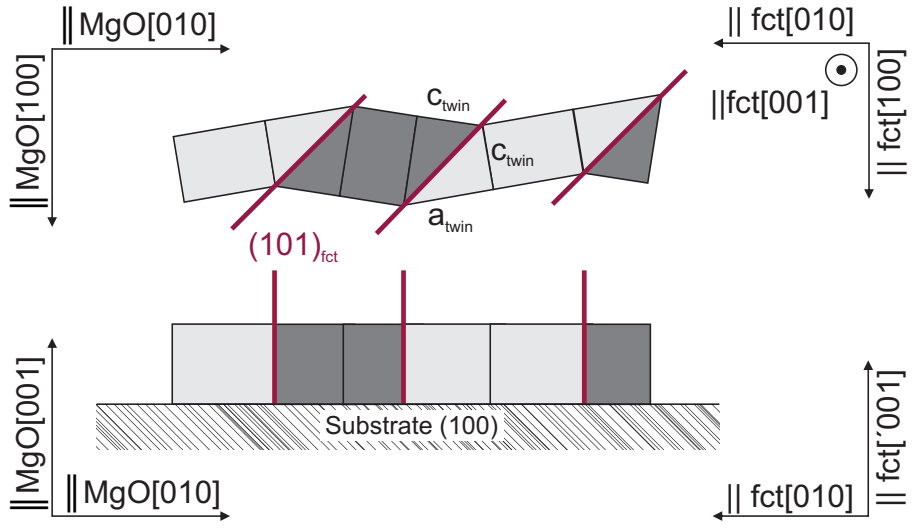

\section{Conclusions}

Commonly, one distinguishes twinning modes by their different origin. Deformation twins are created by external straining, and transformation twins occur during spontaneous straining of the crystal lattice during a martensitic transformation. Growth twins occur during film growth, which often involves both a straining with respect to the substrate and a continuous induction of stress during further film growth. From the crystallographic point of view, however, it is more practicable to use the concept of bcc- and fcc-type twin modes, which allows characterizing twinning within the Bain path and beyond.

We demonstrated the suitability of this approach by analyzing snapshots of Fe-Pd films, where the $c /\left.a\right|_{\text {fct }}$-ratio can be varied over a broad range. As epitaxial films have an absolute reference frame, we could use integral pole figure measurements as a statistically relevant method to confirm this concept.

One key result is that in the as-deposited Fe-Pd films, deformation/growth twins exist. In both cases, the $\mathrm{Fe}_{57} \mathrm{Pd}_{43}$ film on $\mathrm{MgO}$ and the $\mathrm{Fe}_{70} \mathrm{Pd}_{30}$ film on a Cr buffer layer, high compressive stresses lead to the corresponding twin systems, $\{111\}_{\mathrm{fcc}}\langle 11 \overline{2}\rangle_{\mathrm{fcc}}$ fcc-type and $\{112\}_{\mathrm{bcc}}\langle 11 \overline{1}\rangle_{\mathrm{bcc}}$ bcc-type twin systems, respectively. For the $\mathrm{Fe}_{57} \mathrm{Pd}_{43}$ film exhibiting a fct structure, the associated shear of fcc-type twins is 
higher than for bcc-type twins. For the opposed border system, here, the $\mathrm{Fe}_{70} \mathrm{Pd}_{30}$ film on a $\mathrm{Cr}$ buffer with a bct structure, the shear for the fcc-type twin vanishes. However, the shear of the bcc-type twin system is close to the maximum. Such twin boundaries, like the $\{111\}_{\text {fcc }}\langle 11 \overline{2}\rangle_{\text {fcc }}$ mode, are observed in fcc metals with low stacking fault energy during deformation or even film deposition [5,36]. Since deformation and growth twins are crystallographically identical, we cannot distinguish them ex situ. On the one hand, we use PLD for thin film preparation with its high kinetic energy [37] and a low substrate temperature. In combination with a high deposition rate, this would lead to growth twins. Zhou and Wadley [38] used phase field models to simulate the evolution of $\{111\}_{\text {fcc }}$ growth twins. This model indicated that the twin structure is not directly controllable by either the processing temperature or the deposition rate. On the other hand, the Fe-Pd unit cell is tetragonal distorted $\left(c /\left.a\right|_{\text {fct }}=0.97\right)$, which leads to high stresses [28] and, thus, could favor deformation twins.

Furthermore, a consistent description of the orientation relation observed during the martensitic transformation from fcc to fct/bct is presented. Using the phenomenological theory of martensite, the twinned microstructure can be classified with $\{101\}_{\mathrm{fct}}\langle\overline{1} 01\rangle_{\mathrm{fct}}$ transformation twins. This theory is based on one martensitic transformation, using lattice parameters for the austenite and for the martensite. Nevertheless, during annealing, the film should be fcc. After cooling, the austenite reversibly transforms into the fct martensite and at lower temperatures, irreversibly into the bct. However, the rigid interface with the $\mathrm{MgO}$ substrate allows only the formation of variants, with their twin boundary perpendicular to the surface. Using the Bain concept, one can describe this transformation by a continuous change of the tetragonal distortion $\left(c /\left.a\right|_{\text {fct }}\right.$-ratio), which would be contradictory to the martensitic transformation using the PMTC theory. It may therefore be that the Fe-Pd films do not show a real (inter)martensitic transformation.

Finally, an experimental validation for the computational study [17] that suggested adaptive nanotwins in highly strained films beyond the limits of the Bain path is performed. Straining beyond the Bain path opens a new relaxation mechanism in which a single variant with $c /\left.a\right|_{\text {fct }}>1$ spontaneously transforms to a nanotwinned structure with $c /\left.a\right|_{\text {fct }}<1$, exhibiting a lower energy, since they are closer to the global minimum at bcc. The identified low twin boundary energy compared to a high elastic energy is the basis for this nanotwinning. In the present case, a transformation between two tetragonal structures without reducing the symmetry occurs. The artificial (001) film-buffer interface acts as the habit plane.

Since we discuss and provide ex situ results of thin Fe-Pd films, it is not possible to determine the origin of the observed twins. For investigations of the atomic configuration at twin boundaries and the interface to the substrate, transmission electron microscopy is necessary for understanding the dislocation arrangements and the observed morphologies in detail, as it was done for ferroelastic films $[39,40]$.

\section{Acknowledgments}

The authors thank J. Buschbeck, I. Lindemann, A. Backen, A. Diestel, C. Behler and D. Geissler for discussions and experimental support and the German Research Foundation for funding via the Priority Program SPP 1239 and FR 1714/5-1. 


\section{Conflicts of Interest}

The authors declare no conflict of interest.

\section{References}

1. Mahajan, S. Critique of mechanisms of formation of deformation, annealing and growth twins: Face-centered cubic metals and alloys. Scr. Mater. 2013, 68, 95-99.

2. Narita, N.; Takamura, J. Deformation twinning in fcc and bec metals. Dislocations Solids 1992, 9, 135-189.

3. Christian, J.W.; Mahajan, S. Deformation twinning. Progr. Mater. Sci. 1995, 39, 1-157.

4. Kauffmann, A.; Freudenberger, J.; Geissler, D.; Yin, S.; Schillinger, W.; Sarma, V.S.; Bahmanpour, H.; Scattergood, R.; Khoshkhoo, M.S.; Wendrock, H.; et al. Severe deformation twinning in pure copper by cryogenic wire drawing. Acta Mater. 2011, 59, 7816-7823.

5. Lu, L.; Shen, Y.F.; Chen, X.H.; Qian, L.H.; Lu, K. Ultrahigh strength and high electrical conductivity in copper. Science 2004, 304, 422-426.

6. Humphreys, F.J.; Hatherly, M. Recrystallization and Related Annealing Phenomena, 2 ed.; Elsevier: Amsterdam, The Netherlands, 2004.

7. Bhattacharya, K. Microstructure of Martensite: Why It Forms and How It Gives Rise to The Shape-memory Effect; Oxford University Press: Cary, NC, USA, 2003.

8. Niemann, R.; Rößler, U.K.; Gruner, M.E.; Heczko, O.; Schultz, L.; Fähler, S. The role of adaptive martensite in magnetic shape memory alloys. Adv. Eng. Mater. 2012, 14, 562-587.

9. Hultgren, R.; Zapffe, C.A. Gamma to alpha transformation in iron alloyed with palladium. Nature 1938, 142, 395-396.

10. James, R.D.; Wuttig, M. Magnetostriction of martensite. Philos. Mag. A 1998, 77, 1273-1299.

11. Cui, J.; Shield, T.W.; James, R.D. Phase transformation and magnetic anisotropy of an iron-palladium ferromagnetic shape-memory alloy. Acta Mater. 2004, 52, 35-47.

12. Bain, E.C. The nature of martensite. Trans. Am. Insitute Min. Metall. Eng. 1924, 70, 25-46.

13. Buschbeck, J.; Opahle, J.; Richter, M.; Rößler, U.K.; Klär, P.; Kallmayer, M.; Elmers, H.J.; Jakob, G.; Schultz, L.; Fähler, S. Full tunability of strain along the fcc-bcc Bain path in epitaxial films and consequences for magnetic properties. Phys. Rev. Lett. 2009, 103, 216101-216101.

14. Pitteri, M.; Zanzotto, G. Continuum Models for Phase Transitions and Twinning in Crystals; Chapman \& Hall/CRC Press LLC: Boca Raton, FL, USA, 2003.

15. Bilby, B.A.; Crocker, A.G. Theory of crystallography of deformation twinning. Proc. R. Soc. Lond. Ser. A 1965, 288, 240-255.

16. Kurdjumow, G.V.; Sachs, G. Über den Mechanismus der Stahlärtung. Zeitschrift für Physik 1930, 64, 325-343.

17. Kauffmann-Weiss, S.; Gruner, M.E.; Backen, A.; Schultz, L.; Entel, P.; Fähler, S. Magnetic nanostructures by adaptive twinning in strained epitaxial films. Phys. Rev. Lett. 2011, 107, 206105-206105.

18. Hull, R.; Bean, J.C. Misfit dislocations in lattice-mismatched epitaxial films. Crit. Rev. Solid State Mater. Sci. 1992, 17, 507-546. 
19. Oshima, R.; Muto, S.; Fujita, F.E. Initiation of fcc-fct thermoelastic martensite-transformation from premartensitic state of Fe-30at percent-Pd alloys. Mater. Trans. JIM 1992, 33, 197-202.

20. Buschbeck, J.; Lindemann, I.; Schultz, L.; Fähler, S. Growth, structure, and texture of epitaxial $\mathrm{Fe}_{100-x} \mathrm{Pd}_{x}$ films deposited on $\mathrm{MgO}(100)$ at room temperature: An X-ray diffraction study. Phys. Rev. B 2007, 76, 205421-205421.

21. Kühnemund, L.; Edler, T.; Kock, I.; Seibt, M.; Mayr, S.G. Epitaxial growth and stress relaxation of vapor-deposited Fe-Pd magnetic shape memory films. New J. Phys. 2009, 11, 113054-113054.

22. Buschbeck, J.; Hamann, S.; Ludwig, A.; Holzapfel, B.; Schultz, L.; Fähler, S. Correlation of phase transformations and magnetic properties in annealed epitaxial Fe-Pd magnetic shape memory alloy films. J. Appl. Phys. 2010, 107, 113919-113919.

23. Bhadeshia, H. Worked examples in the Geometry of Crystals. Insitute of Metals: London, UK, 2001.

24. Christian, J.W. Accommodation strains in martensite formation, and the use of a dilatation parameter. Acta Metall. 1958, 6, 377-379.

25. Roytburd, A.L. Elastic domains and polydomain phases in solids. Phase Transit. Multinatl. J. 1992, 45, 1-33.

26. Müllner, P.; King, A.H. Deformation of hierarchically twinned martensite. Acta Mater. 2010, $58,5242-5261$.

27. Kaufmann, S.; Niemann, R.; Thersleff, T.; Rößler, U.; Heczko, O.; Buschbeck, J.; Holzapfel, B.; Schultz, L.; Fähler, S. Modulated martensite: Why it forms and why it deforms easily. New J. Phys. 2011, 13, 053029-053029.

28. Edler, T.; Buschbeck, J.; Mickel, C.; Fähler, S.; Mayer, S.G. Mechanisms of stress generation and relaxation during pulsed laser deposition of epitaxial Fe-Pd magnetic shape memory alloy films on MgO. New J. Phys. 2008, 1, 063007-063007.

29. Suzuki, H.G.; Tanino, M.; Aoki, K. Deformation twin in single crystals of a $3 \%$ vanadium-iron alloy. Jpn. J. Appl. Phys. 1966, 5, 879-885.

30. Gruner, M.E.; Entel, P. Impact of local lattice distortions on the structural stability of Fe-Pd magnetic shape-memory alloys. Phys. Rev. B 2011, 83, 214415-214415.

31. Bechtold, C.; Buschbeck, J.; Lotnyk, A.; Erkartal, B.; Hamann, S.; Zamponi, C.; Schultz, L.; Ludwig, A.; Kienle, L.; Fähler, S.; Quandt, E. Artificial single variant martensite in freestanding $\mathrm{Fe}_{70} \mathrm{Pd}_{30}$ films obtained by coherent epitaxial growth. Adv. Mater. 2010, 22, 2668-2671.

32. Oshima, R. Successive martensitic transformations in Fe-Pd Alloys. Scr. Metall. 1981, 15, 829-833.

33. Thomas, M.; Heczko, O.; Buschbeck, J.; Schultz, L.; Fähler, S. Stress induced martensite in epitaxial Ni" Mn" Ga films deposited on MgO(001). Appl. Phys. Lett. 2008, 92, 192515-192517.

34. Kauffmann-Weiss, S.; Hamann, S.; Gruner, M.E.; Schultz, L.; Ludwig, A.; Fähler, S. Enhancing magnetocrystalline anisotropy of the $\mathrm{Fe}_{70} \mathrm{Pd}_{30}$ magnetic shape memory alloy by adding $\mathrm{Cu}$. Acta Mater. 2012, 60, 6920-6930.

35. Khachaturyan, A.G.; Shapiro, S.; Semenovskaya, S. Adaptive phase formation in martensitic transformation. Phys. Rev. B 1991, 43, 10832-10843. 
36. Anderoglu, O.; Misra, A.; Wang, H.; Ronning, F.; Hundley, M.; Zhang, X. Epitaxial nanotwinned $\mathrm{Cu}$ films with high strength and high conductivity. Appl. Phys. Lett. 2008, 93, 083108-083108.

37. Krebs, H. Pulsed Laser Deposition, Applications-Led Growth of Functional Materials; Jogn Wiley and Sons: Hoboken, NJ, USA, 2007; p. 363.

38. Zhou, X.W.; Wadley, H.N.G. Twin formation during the atomic deposition of copper. Acta Mater. 1999, 47, 1063-1078.

39. Romanov, A.; Pompe, W.; Speck, J. Theory of microstructure and mechanics of the ...a(1)/a(2)/a(1)/a(2)... domain pattern in epitaxial ferroelectric and ferroelastic films. J. Appl. Phys. 1996, 79, 4037-4049.

40. Speck, J.; Pompe, W. Domain configuration due to multiple misfit relaxation mechanisms in epitaxial ferroelectric thin-films. 1. Theory. J. Appl. Phys. 1994, 76, 466-476.

(c) 2013 by the authors; licensee MDPI, Basel, Switzerland. This article is an open access article distributed under the terms and conditions of the Creative Commons Attribution license (http://creativecommons.org/licenses/by/3.0/). 